\title{
Doctoral Training for Practitioners: ADAPTr (Architecture, Design and Art Practice Research) a European Commission Marie Curie Initial Training NeTWORK
}

Richard Blythe and Marcelo Stamm

This project has received funding from the European Union's Seventh Programme for Research, Technological Development and Demonstration.

Funding provided under Grant Agreement № 317325.

www.ADAPT-r.eu

The aim of the European Union Marie Curie Initial Training Network Grant 'Architecture Design and Art Practice research' (ADAPTr) is to mobilise the adoption of a practice approach to doctoral research training, and enable seven European universities to: explore its applicability to their contexts; adopt this proven approach to doctoral training through the establishment of an open supervisory network; develop allied support resources; achieve substantial engagement of SMEs (small and medium enterprises) in doctoral training; conduct research into this innovative approach (Blythe et al., 2013). The core feature of this approach to doctoral training is the premise that new knowledges are developed through certain kinds of creative practice (identified as 'venturous') from which research can be 'extracted'. [1] ADAPTr provides a model that fills an internationally identified gap in postgraduate training. It involves reflection on an existing body of peer-acknowledged work and reflection during the production of new works produced concurrently with and informing the doctoral research. The works, processes and methods of the practice are placed in a broader disciplinary context which allows the research to be theorised from within the practice discipline and to clearly identify contributions to disciplinary knowledge. Thus, the research work of the PhD examines, and is also transformative of, the practice within which it is situated. This chapter will provide an overview of this approach to doctoral training with specific reference to ADAPTr and 
the grant's key research work packages.

ADAPTr draws on recent work at RMIT University in developing a design practice research PhD. . Although the first $\mathrm{PhD}$ graduation in this mode was in 2002 the model was originally based on a post-professional masters degree that developed over a twenty year period with significant international input under guidance of Professor Leon van Schaik. [2] In 2011 Professor Richard Blythe further developed the model as the successful basis for ADAPTr and the approach has continued to be refined through the expanded ADAPTr and RMIT teams. [3]

The new paradigm is the result of a careful evaluation of the experimentation undertaken at RMIT University ; it is essentially a carefully constructed and curated social learning model which franchises both SME practices and academic institutions. In it candidates are not treated as isolated individual practitioners but integrated into a research community made up of academics and venturous practitioners. The organisation and choreography of key events are key to the successful open supervisory model in which, while candidates work with a primary supervisor, all supervisors and candidates are continually exposed to and engaged with the work of each other.

The grant activities are focussed around RMIT's two annual Practice Research Symposia (PRS). The practice research symposium is set up as a semi-public event, including the examination sessions. The examinations are by viva and take place within a PhD exhibition. The structure of the symposia sessions and examination process promotes immediate dissemination and contextualisation of the work among a community of scholars. The examinations, coupled with the symposia make it possible to see the entire doctoral process from end to end in one event, an excellent training opportunity for researchers. Further training components use these various activities as an immersive learning platform. A web portal is currently under development that will provide training resources, open courses and a community platform to augment the training aspects of the PRS. [4]

The examinations immediately precede, in-progress review sessions which take place over the PRS weekend. Each candidate has one hour divided into two $25 \mathrm{~min}$ (with a 10 minute set up and transition time). During the first 25 minutes the candidate provides an update and overview of the research as it is progressing, including accounts of key findings and problems. The second session is devoted to discussion with the appointed panel and the wider audience of peers. The panel is appointed by the supervisor and includes the supervisor and other attendees (including examiners) of the PRS event that the supervisor feels will be of benefit to the research. Panels of around 4 are the most effective. An important variation on this model occurs with candidates who are partners in a practice jointly undertaking the PhD. In these 
cases more than one candidates present in a combined session of extended time frame. The decision to present separately or jointly is made between the supervisors and candidates. For the examination multiple approaches have been formulated. In some cases (Kalinina and McAdam) two candidates make independent presentations based on a common exhibition. In the case of fashion designers S!X (Sprynskyj and Boyd), each partner participated in the presentation of the exhibition as a silent assistant which provided the audience with a view of crucial non-verbal interactions between partners of a creative practice.

Candidates are selected into the programme through a pre-application process. Applicants are invited to attend a PRS and to make a 20 minute pre-application presentation in which the applicant has 15 minutes to provide an overview of their practice and insight into its key motivations with a 5 minute window for discussion with a panel of experienced supervisors. The purpose of the presentation is to establish if the practice is substantial enough to sustain a PhD enquiry using this particular methodology.

In addition to the examinations and in-progress reviews the PRS offers an opening keynote lecture which is intended to offer insights into wider research contexts.. Invitees have included recently completed candidates, examiners, and key researchers. The least successful lectures have been from people with no prior engagement with the PRS ecosystem. The weekend concludes with a plenary session in an informal setting in which participants are invited to offer reflections on the PRS, used both in the PRS generally and in ADAPTr as an important quality feedback mechanism and as part of the ongoing review and refinement process.

The PRS is punctuated with informal social activities - morning and afternoon tea, lunches, dinners etc including one formal PRS dinner, which are all critical to the learning model. Many completing candidates report key conversations from these social activities in which colleagues have offered important insights to the research. The PRS model has found resonance with key industry sectors and has been described by one prominent journal as a 'world-leading PhD program for practitioners' in an article in which many of these aspects of the PRS are explained (Rattenbury, 2015).

The four million Euro ADAPTr program is based on forty fellowships, thirty three for 'Early Stage Researchers' (ESR), and seven for 'Experienced Researchers' (ER). The ESR fellowships vary between three and eighteen months and the ER fellowships between twelve and twenty months. While the ESRs are practitioners researching into their own practices, the ERs conduct research across the program at post-doctoral level, researching practice-based research and researching into the research of the ERs. One of the challenging aspects of ADAPTr is that the ESRs are generally more 
senior disciplinary figures than the ERs even though the ERs are more experienced in research conventions, thus inverting the experience and authority that would be more common to these roles. Early results show that the work of the ESRs has provided rich material for researching and that the research of the ERs has been beneficial to the ESR practitioners both in research and in practice terms.

There are four main work packages; Primary Research, Training, Dissemination and Management. The fellows are responsible for the Primary Research deliverables and are active participants in the Training and Dissemination activities. This chapter will focus on the Primary Research work package deliverables.

The Primary Research package is divided into six elements; Case Studies, Communities of Practice, Transformative Triggers, Public Behaviours, Tacit Knowledge, and Methods. These elements were developed based on close observation of the PhD process at RMIT and are significant to practice-based research and effective as supervisory cues for assisting candidates throughout their creative practice research trajectory, but also provide a set of useful perspectives useful for the examination of practice-based research across a collection of individual practitioners. Within ADAPTr the elements are used as scaffolding to guide the PhD work of ESRs who are invited to respond to them in their PRS presentations and also by the ERs who have been researching into them collecting data from the ESRs and other researchers in the PRS ecosystem through interviews, workshops, observing PRS presentations and reviewing documents and works produced by the ERs.

The scaffolding terms of the ADAPTr framework have emerged organically from the practice-based research paradigm; they were either in current use (Public Behaviours, Community of Practice), or which were coined specifically for ADAPTr (Transformative Triggers, Case Studies, Explication of Tacit Knowledge and of Methods). Either way such scaffolding terms have grown from observations of practice-based design research in action for the purpose of exposing gaps in the researcher's description of a practice and the practice itself, for situating the research in relation to its domain, and for identifying ascension moments in a practice. The set of terms is neither absolute nor closed. The domain of practice-based design research is thus not unified by generic and objectified methods of investigation, but rather defined by highly context-dependent, situated, singular and concrete approaches which nonetheless benefit from forms of scaffolding which also provide common points of reference. 

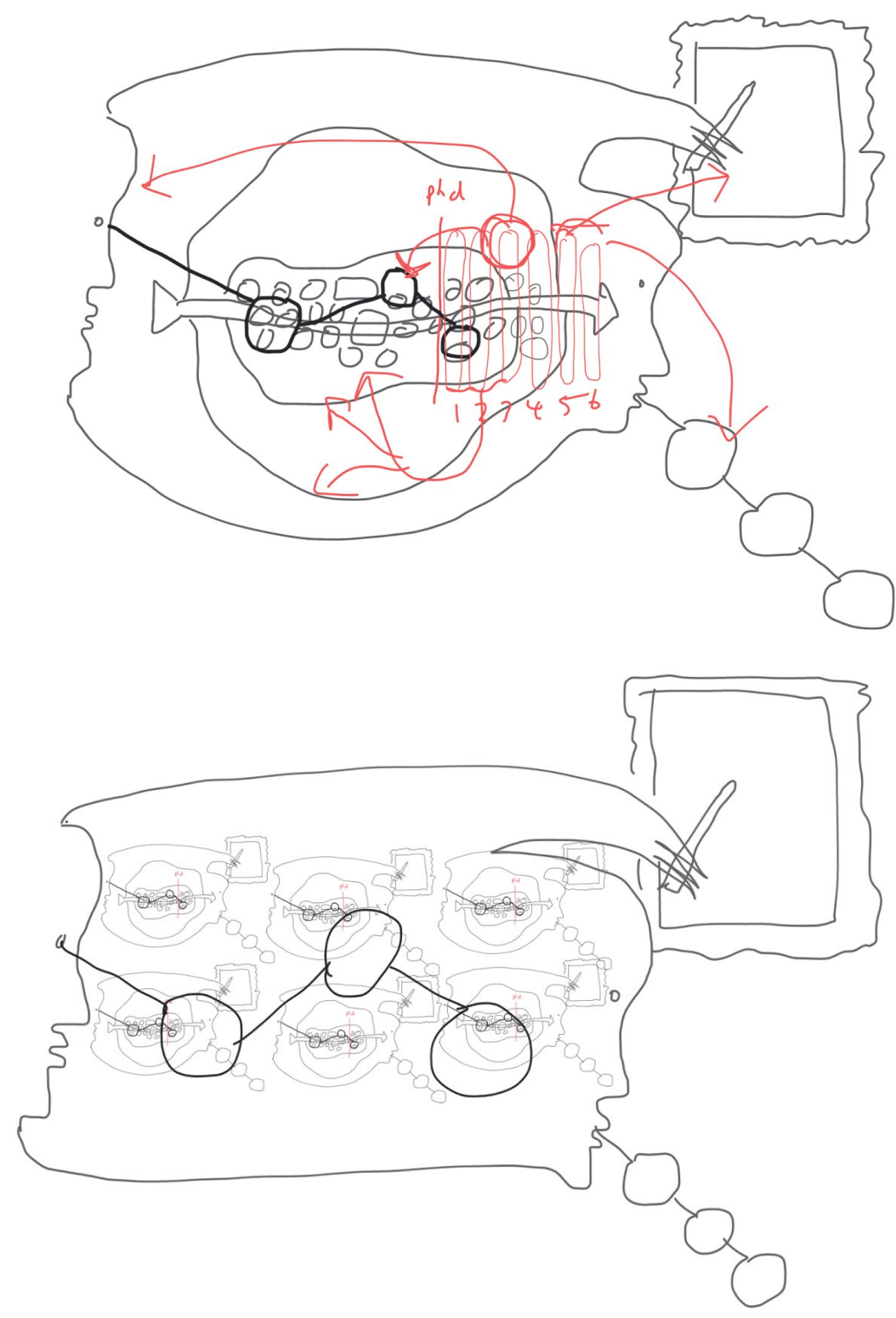

Figure 1. See htps//vimeo.com/112465233. The practitioner reflects on a body of work (the cluster of small circles identifies the series of projects that make up the practice) and works in progress within the context of a wider community of practice (disciplinary context identified by the middle enclosing ring) noting key intentions and ascension moments in the research (identified by the three circles linked to the eye of the researcher). The researcher practitioner then makes an account of this which is communicated through exhibition (picture frame on the top right), verbal presentation (three linked circles falling from the mouth) and exigisis. The PhD is structured around a three year program comprising six PRS events. The numbered lozenges identify each PRS and the arrows point to the foci of each PRS. (Blythe 2014c)

Figure 2. See hitos://himeo com/112465233. This diagram shows a researcher making observations across a range of practices, noting key incidents (three circles linked to the eye of the researcher) and explicating them to a wider audience (same three linked circles now falling from the mouth and through exhibition indicated by the picture frame top right). When viewed en-mass each PhD provides a case study in a wider disciplinary field. This perspective allows for disciplinary or ' $33^{\text {rd }}$ order' insights in which key characteristics can be identified and explicated Blythe 2014b). Supervisors and the ADAPT $r$ ER fellows have undertaken this kind of research but each PhD candidate also, to some extent, operates at this order in locating their research/practice within the disciplinary field. (Blythe 2014c)

Case Studies in Practice Based Design Research

The term 'case study' in practice based design research refers to an individual practitioner's comprehensive research into his practice: each practitioner as such represents a singular case study. The specificity and singularity of the case study in question must not be misinterpreted as suggesting a purely subjective nature of the case under scrutiny. It is by virtue of the situated, concrete, embodied and materialised nature of practice that observations are made that can be shared as they can be shown and used as communal points of reference by different researchers.

The notion of the practitioner's individual case study can be related to the idea of three orders of knowledge to be 
distinguished with regard to the way a practitioner contextualises his work: Specific select projects, potentially grouped under certain research relevant perspectives ( $1^{\text {st }}$ order of knowledge) can be contextualised within the full body of work of the practitioner, so that e.g. transformations and shifts in the practice may become apparent ( $2^{\text {nd }}$ order of knowledge). In a transversal perspective, the individual case study of one practitioner may then be differentiated and positioned within a field of parallel, adjacent or contrasting other case studies ( $3^{\text {rd }}$ order of knowledge) (Blythe 2014b).

\section{Communities of Practice}

The investigations of a practitioner's community of practice serves a two-fold purpose: to contextualise and position an individual research case study within a realm of practices for which the findings and outcomes of the research in question may have relevance and impact, but also in order to reference the practice into adjacent practices that may exhibit different degrees of influence on the practice under scrutiny. Due to the critical nature of situating the research in the field, the notion of 'community of practice' is an instrumental term that allows the researcher to potentially differentiate one's own research from that of seemingly neighbouring peers: divergence and dissent become as important as possible confluence and resonance. The practice which is conducted in research mode may conceive a community of practice also as a critical 'community of inquiry'.

Rather than conceiving communities of practice as a static stock of backdrop practices, they are highly dynamic and varied to the point where the researcher may attribute variable communities of practice to different individual projects within the body of work. Communities of practice may be also understood in a narrow sense as the aggregation of contributors that play a role in the production of a piece of work by way of direct collaboration with the practitioner undertaking the research.

A community of practice can be understood as the choir of voices that operates as self-talk during the creative process. This self-talk, although an individual phenomenon, is an internal conversation with peers and collaborators, (and sometimes challengers) extending the social aspects of community also to individual thinking.

\section{Transformative Triggers in Practice based design research}

The notion 'transformative triggers' serves to induce reflection both into the dynamic and possible transformation of a practice and into the roots and causes for potential shifts and changes observed in a practice. A specific explanatory gap identified during the research process can 'trigger' a new project that interrogates the bespoke issue in order to close the 
particular research gap through a transformed practice in response to such gaps.

Some researchers distinguish between tranches of work that reflect transformations of the practice and respective phases triggered by shifts in perspective, in approach, attitude, intended design outcome. Practice-based design research thus uses the term transformative triggers to sensitise the researcher towards detecting and analysing both transformations and transformational stimuli at different levels and phases of the practice and in different orders of knowledge under scrutiny.

\section{Public Behaviours in Practice based design research}

The term 'Public Behaviours' stems from close observation of the 'natural history' of the creative individual in general who seeks rhapsodic exposure to public scrutiny and validation of the quality and innovative nature of the practice, but then retreats again into the non-public sphere of the individual practice laboratory where new observations and discoveries are made which may innovate and transform the practice afresh.

Such public behaviour follows a repeated pattern and propensity to seek recognition of claims and perceived achievements of a venturous practice in a public realm. Public 'practice behaviour' platforms include actual and concrete realms of exposure such as client relations, exhibition, competition and award fora; it can also consist in virtual fora such as new social media or communities 'at a distance'. Specific platforms for public 'research behaviour' include the two-yearly Practice Research Symposium (PRS), but also the standard peer review process associated with research publication outlets.

A specific sense of 'public' behaviour is linked to the idea of the practitioner's enchainments: creative and innovative achievements can be traced back to specific forms of constellational engagement of the practitioner with other individuals and external positions; such an engagement in turn depends of the practitioner's capacity to constellate - i.e. to be able to operate in public modes beyond the stereotypes of the notoriously secluded and solitary endeavour of the creative individual.

Tacit Knowledge in Practice based design research

The notion of tacit knowledge points towards dimensions of something 'known' but not articulated, as well as to specific modes of 'knowing' akin to 'experiential knowledge' and 'operational knowledge' that can be shown, exhibited and demonstrated, rather than made explicit in discursive and conceptual ways. The practitioner thus may also explore individual 
forms of explicating modes of 'knowing how' rather than 'knowing that'. The practitioner observers herself as operating on the basis of knowledge dimensions and resources that are not or cannot all be at the same level of articulation. The sister notion of 'embodied knowledge' also points at 'material knowledge' and materiality and the body as 'sites of knowledge' in the wake of understanding thinking as potentially material and intelligence embodied in morphology and materiality rather than dependent on and residing in a conceptual realm.

Practice-based design research understands that there is no singular realm of 'tacit knowledge', but that the 'tacit' relates to diverse layers and dimensions of the unspoken, including what the practitioner takes for granted or regards as trivial. To foreground, uncover, expose, unfold and extract what is otherwise neglected, covered up, avoided or compacted can take the form of (self-)critical engagement with what is wrongly assumed, with unreflected presuppositions and pre-conceptions, hidden premises, (cognitive) biases operational routines and 'thinking styles'. Under the critical lens, the tacit points towards a disjunct between what the practitioner (sometimes mistakenly) perceives prima facie as central to the practice and what may be operative in the background; tacit knowledge analysis may thus help to debunk myths regarding the principles of operation in a practice.

\section{Methods in Practice based design research}

Practice-based design research cannot draw upon a received and sanctioned set of research 'methods': the term, while it suggests that some such repertoire of methods may be available, is therefore contested: Practitioners sometimes prefer to talk about individual research strategies and specific research tactics on the intentional side (e.g. 'mapping', 'categorising') and to particular research techniques on the operative side (e.g. 'drawing', 'diagramming', 'videoing' etc.).

It is a basic premise of practice-based design research that each practitioner has to develop and test a distinctive individual range of ways to conduct the research. While there may be common and shared perspectives and useful lenses (or temporary scaffolding) that suggest themselves within the practice-based paradigm, each researcher will have to develop and explore an individual methodological take on how to investigate these possible research perspectives. Different directions and foci of reflection warrant different ways of conducting such diverse reflections.

Methodological approaches are individualised through the application of techniques that have been developed within the ecology of the specific practice and specific to the discipline. The advancement of the discipline through the explanation of methods depends not on imitation, repetition and literal transfer of methods in formulaic patterns, but on ongoing adaptation, mutation and recasting of approaches as they are explained and explicated in their dependency on new 
research contexts and projects. The specific adequacy of a method, its relevance and yield are then understood by the fellow practitioner as belonging to a specific practice and interrogation. It is only by virtue of methodological explicitness at this level of specificity that 'methods' in practice based design research can claim a second order transferability and objectivity.

In the same way as a case study may mature to the point where the practitioner's highly individual and authentic voice emerges and generic jargon and abstract discourse recede, an authentic mode of researching emerges as a genuine research achievement in its own right.

\section{Conclusion}

The ADAPTr work package concepts have been developed, and continue to develop based on close observation of the ways in which creative practitioners have gone about the extraction of a $\mathrm{PhD}$ from their design practice,adapting and refining design practice approaches and methods to research. This kind of research is founded in and continually 'sheeted' back to practice through careful observation. As Marcelo Stamm has described (2015), this specific research paradigm is rooted in material and practice knowledge. Stamm is cautious of concepts external to design disciplines. For example at the ADAPT_r Making Research Researching Making 2015 conference keynote speaker Albena Yaneva described how in her recent collaboration with Alejandro Zaera Polo, she had applied a philosophical concept - cosmopolitical - to a reading of architecture and its practice. While Yaneva's work may provide some insight into the condition of architecture from an external view point (a sometimes useful perspective), it is questionable whether such a perspective provides substantial insight into the practice of architecture itself; a philosophical concept can easily misdirect also in research terms, and force you to look for something that is not there at all (Stamm 2015).

This condition has also been described by Blythe as a 'topological error' in design research (Blythe, 2012). Blythe points to the ways in which different knowledge realms hinge at a certain point, reflect into each other and yet need to be understood as autonomous - meaning that it is common but highly problematic to seek to establish research authority for design disciplines from within other disciplines such as philosophy even though each may reflect usefully into the other in the creation of knowledge. To illustrate this point the position seems almost absurd if expressed in the reverse direction: that philosophy might seek to authenticate itself through art practice - even though art practice may be a productive muse for philosophy.

The ADAPTr work package terms are used in an instrumental supervisory sense to assist design 
practitioner-researchers to find valid means of examining their own practice while avoiding these kinds of common research pitfalls and to provide an open structure (meaning both not a closed set and also open to continual adaptations) to assist in the PhD process for candidates and for supervisors. The PRS structure, the joint supervisory model and the continual invention and refinement of terms through the PRS process shared between candidates and supervisors facilitates an effective way to extract a $\mathrm{PhD}$ from an existing practice which allows the practitioner to remain primarily focussed on practice which is positively transformed through the process. The net result is a the formation of a researcherly network of $\mathrm{PhD}$ trained practitioners who are enabled to operate across both academia and industry in new ways.

References

BLYTHE, R. 2012. Topological Errors in Practice based design research: Understanding the Reflective Hinge and the Reflective Gap. In: BOUTSEN, D. (ed.) Good Practices Best Practices. Antwerp: EPO.

Framework for a Practice Based PhD, 2014a. Directed by BLYTHE, R. Vimeo. https://vimeo.com/90515214

Three Orders of Design Knowledge, 2014b. Directed by BLYTHE, R. Vimeo.

https://vimeo.com/116316562. Keynote address, Melbourne PRS, 22-26 $6^{\text {th }}$ October 2014.

How to do a Practice Based PhD in Three Minutes, 2014c. Directed by BLYTHE, R. Vimeo. https://vimeo.com/112465233

Richard Blythe, Johan Verbeke, Clause Peder Pedersen, Katherine Heron, Tadeja Zupančič, Veronika Valk \& Sally Stuart. 2013. ADAPTr: Architecture, Design and Art Practice Research. Ghent, Barcelona: European Union's Seventh Programme for Research, Technological Development and Demonstration. European Commission, http://cordis.europa.eu/projects/rcn/106609 en.html. www.ADAPT r.eu.

BLYTHE, R. \& SCHAIK, L. V. 2013. What if Design Practice Matters? In: FRAZER, M. (ed.) Design Research in Architecture. Surrey, England: Ashgate Publishing Limited. 
Eli Hatleskog \& Anna Holder. 2015. Transformative Triggers: 10 Individual Accounts of Transformative Practice Triggers. ADAPT_r European Union's Seventh Programme for Research, Technological Development and Demonstration. European Commission.

RATTENBURY, K. 2015. The Imitation Game: the best crit system in the world? International PhD programme PRS lets architects develop their work as research. RIBAJ.

Schaik, Leon van \& Spooner, Michael. 2010. The Practice of Practice 2: Research in the Medium of Design. Melbourne: School of Architecture and Design, RMIT University.

SCHAIK, L. V. 2009. Design Practice Research, the Method. In: VERBEKE, J. (ed.) Reflections. Brussels: St Lucas.

SCHAIK, L. V., WARE, S. A., FUDGE, C. \& LONDON, G. 2014. The Practice of Spatial Thinking: Differentiation Processes, Melbourne, Onepointsixone.

VAN SCHAIK, L. \& JOHNSON, A. (eds.) 2012. The Pink Book. By Practice By Invitation., Melbourne: Onepointsixone.

Leon van Schaik. 2005. Mastering Architecture: Becoming a Creative Innovator in Practice (Architecture in Practice). John Wiley and Sons.

Leon van Schaik. 2003. The Practice of Practice: research in the medium of design. Melbourne: RMIT University Press.

Marcelo Stamm. 2013. Reflecting Reflection(s): Epistemologies of Creative Practice Research. Brussels: St Lucas, University of Leuven.

Marcelo Stamm. 2015. Conditions of Creativity. Researching Creation Through Creation: Nothing is Hidden. Aarhus: ADAPT_r European Union's Seventh Programme for Research, Technological Development and Demonstration. European Commission .

Endnotes

[1] 'Venturous' was originally adapted by Richard Blythe from Terry Cutler's Venturous Australia 
report on research and innovation and has become part of the language of the program distinguishing practices which undertake 'researcherly' practice and those practices that deliver a professional service.

[2] See Three Orders of Design Knowledge, 2014b. Directed by BLYTHE, R. Vimeo. https://vimeo.com/116316562; and Framework for a Practice Based PhD, 2014a. Directed by BLYTHE, R. Vimeo. https://vimeo.com/90515214. Blythe's simple RMIT model video, designed for candidates and supervisors, has had over 10000 loads in more than 100 countries since it was posted publicly on Vimeo in 2014.

[3] For further information on the RMIT program see: SCHAIK, L. V. 2009. VAN SCHAIK, L. et al. (eds.) 2012. BLYTHE, R. et al., 2013. STAMM, M. 2013. SCHAIK, L. V, et al., 2014. STAMM, M. 2015.

[4] The site can be accessed at creativepracticeresearch.info 